\title{
The Art of Forensics, Solving Florida's Cold Cases 2016: Case 5 Study
}

\author{
Paloma Joana Galzi ${ }^{1 *}$, Hannah Isaacs ${ }^{2}$, Tatiana Der Parthogh ${ }^{3}$ \\ ${ }^{1}$ Forensic Imaging Specialist, Galzi Forensics Limited, USA \\ ${ }^{2}$ Forensic Artist, USA \\ ${ }^{3}$ Forensic Artist, Art Loss Register, USA
}

Submission: March 15, 2017; Published: May 03, 2017

*Corresponding author: Paloma Joana Galzi, Forensic Imaging Specialist, Galzi Forensics Limited, 38 Battledean Road, USA, Tel: 07539466653; Email: paloma.galzi@gmail.com

\begin{abstract}
This article will relate to the events that took place in Tampa Florida, in October 2016 at the University of South Florida where the Forensic Anthropology Unit teamed up with qualified Forensic Artists from around the world for one week, to help solve 20 cold cases using Facial Reconstruction techniques as a last resort for identification. Forensic Facial Reconstruction is the process of creating the face of an individual on a skull for the purpose of identifying an unknown person. It has become a powerful tool that significantly enhances the chances of identification of the deceased on skeletal remains. 3D printed skulls where provided to the team of forensic artists by the University of South Florida (USF), and the facial reconstruction process conducted following the anatomical method developed by Gerasimov and adapted by Neave [1], where muscles of the head are to be modeled on the printed 3D skull with Plasticine Clay before applying another layer for the skin. This technique allows a better and more accurate interpretation of the human remains to depict the face of the deceased.
\end{abstract}

Keywords: Facial Imaging; Facial reconstruction; Forensic science; 3D modeling; Anatomical method; Facial Identification; Facial Recognition; Florida; Tampa State Police; NCMEC; University of South Florida

\section{Introduction}

Over the last few decades, Facial Reconstruction has proven to be reliable and successful, and many forensic investigations have used this technique to produce identification. The Anatomical method, which will be explained here, includes modeling the muscles structure before applying the skin to depict the living facial appearance [2]. This method claims a $75 \%$ rate of success with the aim of recreating a face that would resemble the deceased $[3,4]$. For this second annual event of "The Art of Forensics, Solving Florida's Cold Cases", Dr. Erin Kimmerle and her team of forensic anthropologists at the USF Institute for Forensic Anthropology and Applied Science (IFAAS) gathered 20 different cases from all over the United States, some decades old. Six of the cases were sets of remains found in the 1970s, and two were corpses located in 2016. Twelve were found in Florida, two from Kentucky, four from Pennsylvania, and one each from Missouri and Tennessee. Three of the Pennsylvania cases were remains recently exhumed, and were homicides in the 1970s in Luzerne County according to officials [5].

The anthropology team then proceeded to establish a profile for each case (giving an estimation of ancestry, age and sex), in the hopes that through the use of skeletal analysis, facial reconstruction techniques and chemical isotope testing, the deceased will be given their identity back. The 2015 event of Solving Florida's cold cases had led to the positive identification of Jessica Rousseau [6]. The idea of the workshop was developed by Joe Mullins, forensic imaging specialist at the National Center for Missing and Exploited Children for over 16 years, with the help of Dr. Erin Kimmerle, forensic anthropologist, associate director and executive director at the USF Institute for Forensic Anthropology and Applied Science. The workshop, led by Mullins, was developed in the hopes that someone will recognize the deceased people as a last resort for identification. On the last day of the workshop, the reconstructions were presented to the press where artists, speakers and law enforcement where present.

\section{Case 5: Tampa police department}

Using the latest 3D technology available, the forensic artists started to each work on their skull in the lab of Dr. Kimmerle. Upon arrival of the 14 specialists, the lab was equipped for each and one of us with a case report and analysis file, sculpting 
tools, and 3D printed skull of the remains to work on. It is important that we only work on skull replicas for two important reasons: preservation of the skull with a non-invasive method of reconstruction and if possible, to keep the original next to the replica for better reference and accuracy along the way [6]. Case 5 was turned over to the Tampa Police Department as a cold case. In December 1985, human remains were discovered in a remote field used as an unauthorized trash-dumping site in Tampa, Florida. USF's Anthropology team established the remains to be those of a 20-30 year-old petite black female, approximately $5^{\prime} 2$ " to 5'9" tall. She was found nude and her death was ruled as a homicide. Chemical isotope testing was conducted to estimate her geographical origin, which was consistent with local USA origin of birth.

\section{Methodology}

\section{Anatomical process of reconstruction}

The Soviet archeologist and anthropologist Gerasimov pioneered paleo-anthropological facial reconstruction of historic figures. He developed a deep understanding of the relationship between the skull and the facial soft tissues, technique known today as the European method: individual muscles are made from clay and attached to the skull, one by one, according to the origin and insertion of each muscle [7]. The American method suggests that the facial muscles are not modeled, and the face is sculpted using only the tissue depth markers connected by clay strips creating the face contour [4]. A synthesis of these two methods, known as the Manchester Method and developed at the University of Manchester in the UK allows for individual muscles to be attached on the skull while using the tissue depth measurements as a guide of the final architecture of the face [4]. For the purpose of the workshop, all the skulls were previously mounted in the Frankfurt plane, which is reached when a horizontal line passes through the inferior border of the orbit and the external auditory meatus [4] (Figure 1).

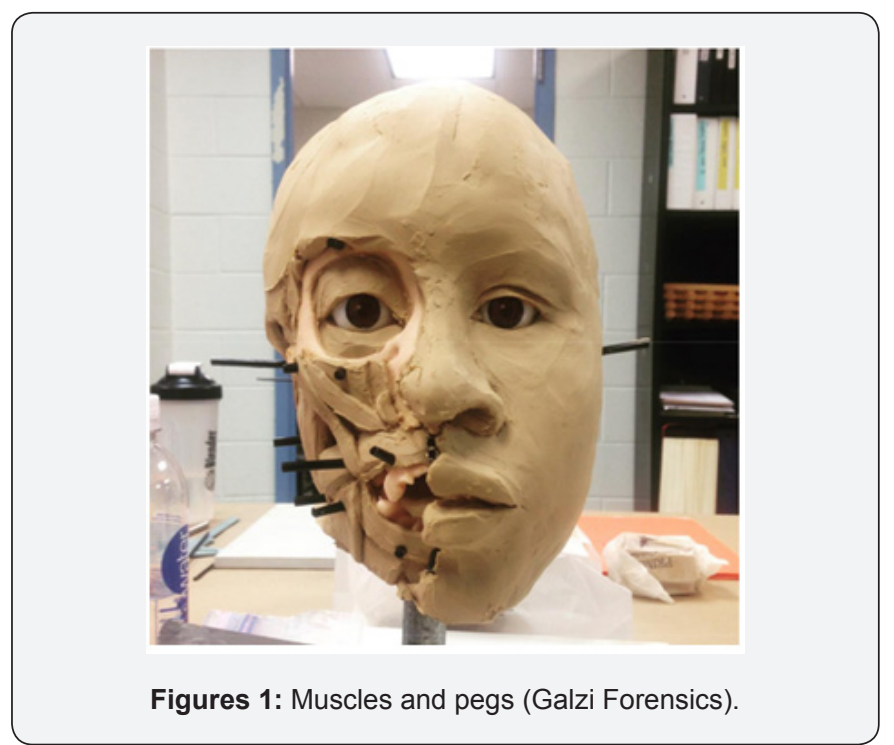

\section{Assessment}

Before any work can be executed, the specialist needs to know certain information about the skull, such as its ancestry, sex determination and age. The specialist usually has access to the anthropology report, which will specify those characteristics that he will be able to compare. Not all skulls received are complete and intact, but for case 5, no parts were missing. The ancestry can be determined by examining the morphology, or specific traits of the skull, that will provide information on a certain ancestral group. On case 5 , it was determined by USF that the remains were that of a black person, which can be shown by a wider distance between the eyes, a low rounded nasal root, a wide nasal aperture, and a facial prognathism more pronounced than remains from the European ancestral group for example.

This will show a more fuller and rounded forehead, a flatter nose, prominent cheekbones, and wider lips $[4,8]$. The sex can be determined by a series of traits that can be observed and are easier to find on a skull if that person has reached maturity [9]. A female skull will generally be rounder, more gracile, and smaller than the male. The orbits will be rounder, with softer brow ridges, less pronounced. The jaw line will tend to be less robust, and rounded as observed as well on the mental protuberance, the occipital one and the mastoid process [4]. Teeth and their growth mainly determine the age from a skull during life [10]. On case 5 , it was established that the black woman was between 20 and 30 years of age at death (Figure 2).

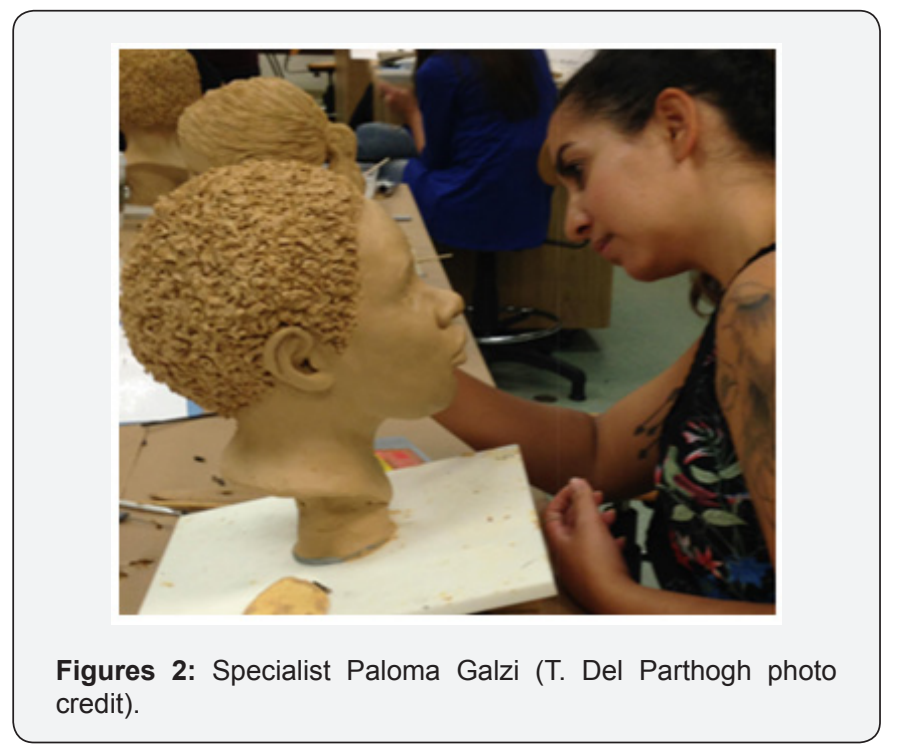

\section{Forensic reconstruction process}

The first step was to model and position the facial muscles onto the skull replica. The Temporalis muscle act as a synergist of the masseter in closing the jaw. The masseter allows movement of the jaw, opening and closing it. The orbicularis Oris is the muscle around the mouth, allowing its movement (the kissing muscle). The Buccinator flattens and compresses the checks, and is a chewing muscle. The Mentalis, Depressor Labii Inferioris 
and Depressor Anguli Oris are muscles connecting the mouth to the chin, allowing movement of the lower lip. The Levator Labii Superioris, and Levator Anguli Oris muscles allow movement of the upper lip. The Nasalis muscles connect the top of the nose to the upper lip, and the Zygomaticus muscles pull the corners of the mouth (Figure 3). The second step was to position the tissue depth markers on Forensics) different osteological landmarks points on the skull. They will indicate the thickness of facial tissue to apply on the skull as a guide. The tissue depths vary between sexes, ancestry and ethnic groups, and consist in little pegs positioned on the skull at a 90 degree angle to the bone surface.

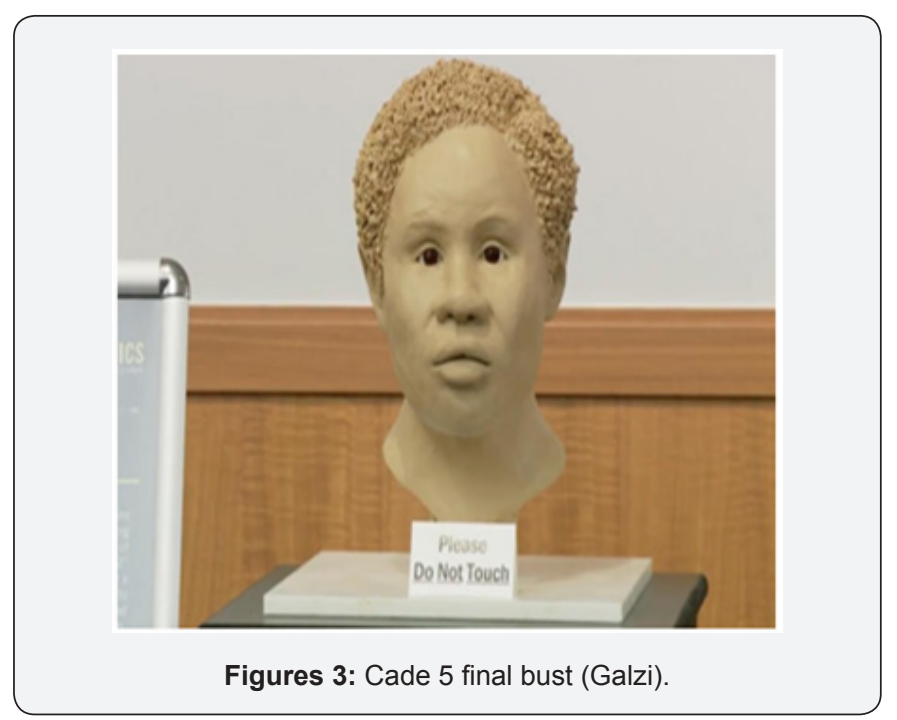

For the workshop, the FBI 2016 tissue depth data was used as an average and pegs measured according to this data. The third step of the process consisted in applying the layer of the skin using the pegs as a thickness guideline, and modeling the facial features accurately [11]. We started with positioning the eyes in their orbits with marble globes (the average eyeball is 24-25mm), taking into consideration that no face is completely symmetrical $[4,12]$ (Figure 4). The globes were centered in the orbits so the eyelids could be correctly placed as well as the eyebrows, following the shape of the superior orbital margin. In 1912, Wilder stated that the inner and outer corner of the eyes were precisely determined respectively by the lacrimal fossa and the malar tubercle, often easy to find on the skull. The nose was determined by calculations of its shape based on the nasal aperture, its angle and its width [11,13], and appeared quite wide and rounded.

The mouth was shaped one lip at the time based on the underlying structure of the bone, in reference to the inter-canine distance and the height of incisors, the occlusion of the teeth, the dental pattern and the shape of the jaw [14-16]. The lower lip did appear fuller than the upper one, and prognathism did show on the profile. The ears were modeled in reference to the angle of the jaw (the ramus) and the shape of the mastoid process indicated the shape of the ear at its base [17]. The hairstyle was done last, as an approximation of the texture that could have been the victims'. Upon finishing the facial reconstructions of all 14 cases from Monday through Thursday of that same week, each and every one of them were photographed in different views (Figure 4) by the USF anthropology team. The busts were then moved to the Tampa Bay History Center to be revealed on the Friday for the event at the "Art in Forensics, Solving Florida's cold cases".

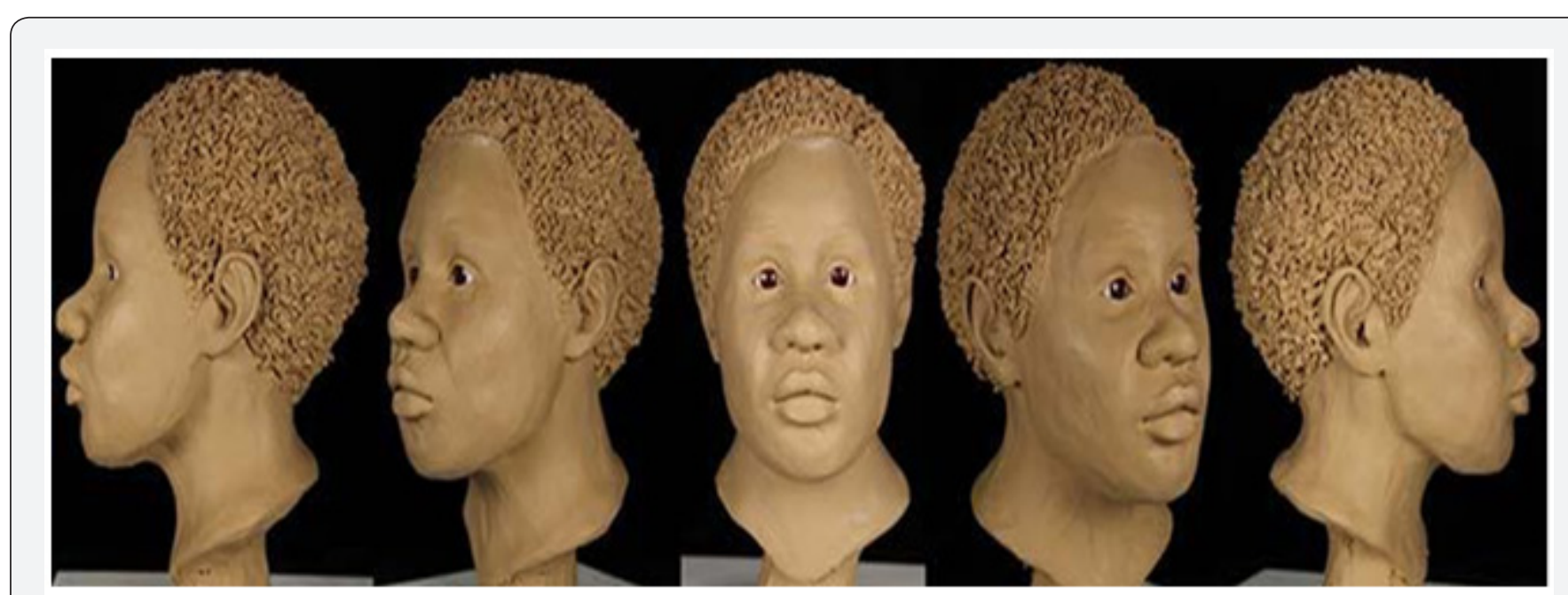

Figure 4: Case 5 views (USF photo credit).

\section{Conclusion}

The work carried out by the specialists over this intense week brings cold cases to light as a last chance for people to recognize the deceased and identify them. This anatomical method of Facial Reconstruction is at low cost for all the parties involved, and has proven to be efficient and reliable over the past few years provided that the practitioner is experienced with a thorough 
knowledge of facial anatomy. In this case, the final event "Art of Forensics: Solving Florida's cold cases" was advertised and open to the general public and press to view the busts, hear about the process and ask questions. Sheila Williams and Sharon Scott had heard of the event and came to see the busts in the hope to find more answers of their long lost sister, who disappeared almost 40 years ago. To them, case 5 was a really close match, finding similarities in the eyes, nose and forehead of the bust. At this time, DNA comparison is being done, and we are hopeful that it will bring this family closure (Figures $3 \& 5$ ).

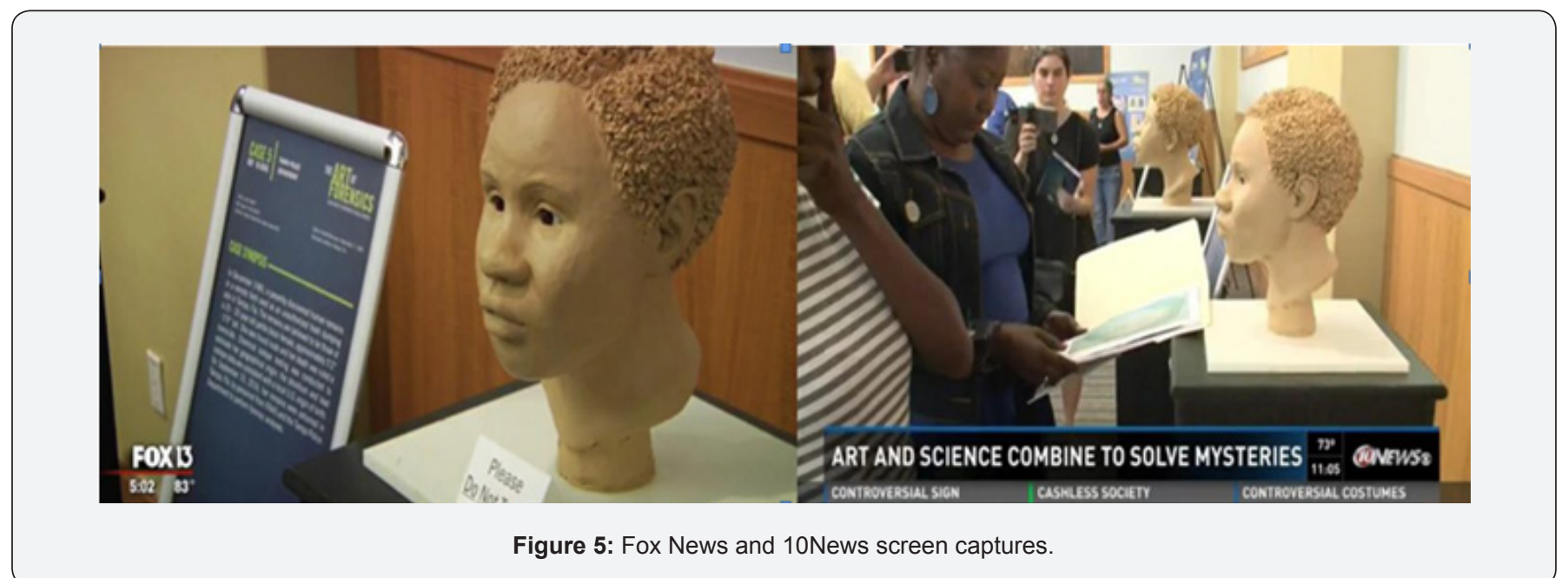

\section{Acknowledgement}

We would like to thank the University of South Florida, Dr. Erin Kimmerle and her anthropology team for setting up the workshop and gathering all those cold cases. With the help of the National Center for Missing and Exploited Children and Joe Mullins, and all the celebrated specialists that attended this event for the second time.

\section{References}

1. Gerasimov (1971) The face finder. Hutchinson, USA.

2. Prag J, Neave RAH (1997) Making Faces. British Museum Press, USA.

3. Wilkinson \& Whittaker (2002) Juvenile forensic facial reconstructiona detailed accuracy study.

4. Wilkinson CM (2004) Forensic Facial Reconstruction anatomical art or artistic anatomy. J Anat 216(2): 235-250.

5. Seth Augenstein (2016) USF workshop forensically recreates cold case faces. Forensic Magazine.

6. Mark Schreiner (2016) Art of Forensics aims to put names to cold case victims. WUSF News, USA.
7. Cooper, Fitzgerald, Mills, Levitt (2008) Forensic facial reconstruction.

8. Krogman, Iscan (1962) The Human Skeleton in Forensic Medicine.

9. Stewart TD (1948) Medicolegal aspects of the skeleton- age, sex, race and stature. American Journal of Physical Anthropology.

10. Schour, Massler (1944) Development of human dentition. Chicago University of Illinois School of dentistry, USA.

11. Black S, Thompson T (2006) Boca, Forensic Human Identification - An Introduction, Ratan: CRC Press pp. 544.

12. Bailey L (2014) Ask a forensic artist.

13. Rynn, Christopher, Wilkinson, Caroline M () Prediction of nasal morphology from the skull. Forensic Sci Med Pathol 6(1): 20-34.

14. P Galzi, J Mullins (2016) Case Study: 3D Application of the Anatomical Method of Forensic Facial Reconstruction.

15. Wilkinson, Rynn (2012) Craniofacial Identification. Cambridge p. 8.

16. Wilkinson CM (2010) Facial reconstruction- anatomical art or artistic anatomy? J Anat 216(2): 235-250.

17. Taylor (2001) Forensic Art \& Illustration. USA, pp. 608.

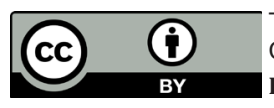

This work is licensed under Creative Commons Attribution 4.0 License DOI: $10.19080 / J F S C I .2017 .03 .555606$

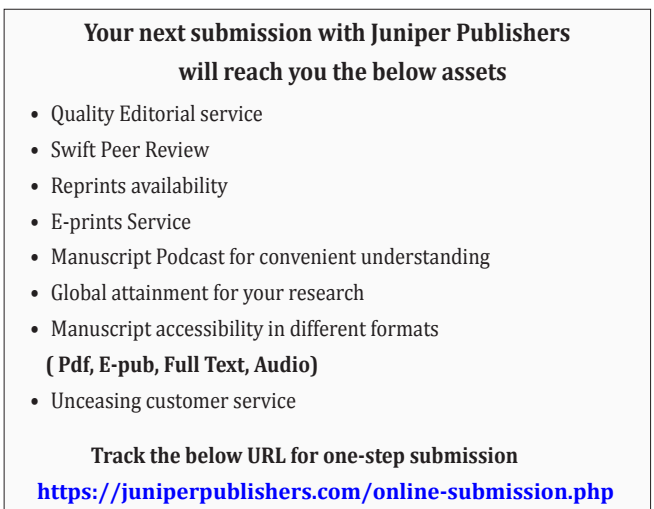

